\title{
3D-printed pelvis model is an efficient method of osteotomy simulation for the treatment of developmental dysplasia of the hip
}

\author{
KEXIN LIU, ZITAO LI, YUBO MA and HONGYU LIAN \\ Orthopedics Surgery Department 2, Affiliated Hongqi Hospital, Mudanjiang \\ Medical University, Mudanjiang, Heilongjiang 157000, P.R. China
}

Received January 18, 2019; Accepted July 12, 2019

DOI: $10.3892 /$ etm.2019.8332

\begin{abstract}
Developmental dysplasia of the hip (DDH) is a congenital or developmental deformation of the hip joint, which may require a high number of surgical interventions. It has been indicated that $3 \mathrm{D}$ printing may be used to simulate a fractured pelvis to facilitate the fixation of plates during the surgical procedure. In the present double-blinded randomized clinical trial, the utility of the 3D-printed pelvis model, comprising 3D reconstruction, reverse engineering and rapid prototyping, in the treatment of DDH was evaluated with 3D $\mathrm{CT}$ as control. The value of the $3 \mathrm{D}$-printed pelvis model in the surgical management and development of a strategy for an individualized operation for DDH using osteotomy simulation was also assessed. The results indicated that use of the 3D-printed pelvis model increased the success rate of the operation with a shortened surgery time and post-operative recovery time for $\mathrm{DDH}$ patients. In addition, the application of the 3D-printed pelvis model allowed for more efficient surgical management of DDH than 3D CT and promoted post-operative recovery of the DDH patients. Pre-operative planning using the 3D-printed pelvis model was feasible for DDH patients. Furthermore, few patients exhibited delayed incision healing, wound infection or nonunion in the DDH group with osteotomy simulation using the 3D-printed pelvis model or 3D-CT. In conclusion, the present study indicated that the 3D-printed pelvis model, including 3D reconstruction, reverse engineering and rapid prototyping, constitutes an efficient tool for pelvic osteotomy simulation, which improves personalized pre-operative planning by providing a visual and accurate osteotomy model for patients with DDH (Chinese Trial Registry No. KCT0012374).
\end{abstract}

Correspondence to: Professor Hongyu Lian, Orthopedics Surgery Department 2, Affiliated Hongqi Hospital, Mudanjiang Medical University, 708 Guanghua Street, Xi'an, Mudanjiang, Heilongjiang 157000, P.R. China

E-mail: hongyu_doctor@126.com

Key words: 3D printing, developmental dysplasia of the hip, osteotomy simulation, pelvis model

\section{Introduction}

Developmental dysplasia of the hip (DDH) is a congenital or developmental deformation or misalignment of the hip joint, and is one of the most common congenital defects in the newborns, which significantly impairs skeletal development in children (1). DDH has become one of the most common types of skeletal congenital anomaly and affects 1 in 1,000 live births in China (2). It has been indicated that DDH may be influenced by environmental and genetic factors and the incidence in females is five times that in males (3). Surgical management is the most common treatment for DDH of severities ranging from a mild form of acetabular dysplasia to a moderate form of subluxation of the hips (4). A systematic review demonstrated that the incidence of avascular necrosis was frequent following medial open reduction in pediatric patients with DDH (5).

At present, surgery is the most commonly used treatment for DDH, and the success depends on the accuracy of the pelvic osteotomy (6). The 3D printing technique has been applied in orthopedics research on trauma, tumor, joint replacement and pedicle screw fixation $(7,8)$. Zheng et al (9) suggested that a 3D-printed navigation template in proximal femoral osteotomy simplifies surgery and improves the precision for older pediatric patients with DDH. In addition, Wu et al (10) indicated that the 3D printing technique provides virtual pre-operative planning prior to reconstruction of old pelvic injuries. Zengy et al (11) suggested that the $3 \mathrm{D}$ reconstruction technique may restore the true acetabular morphology and allows for quantitative analysis of DDH patients. Of note, a pilot study demonstrated the potential application of the rapid prototyping pelvic model for patients with DDH, which facilitates arthroplasty planning and surgical procedures due to better planning and improved orientation (12).

To explore the value of the 3D printing technique in the surgical management and strategy of rehabilitation therapy for DDH, a 3D-printed pelvis model was used as an adjuvant protocol for the DDH surgery, and 3D reconstruction, reverse engineering and rapid prototyping were employed. The present study indicated that osteotomy simulation using the 3D-printed pelvis model contributes to the surgical management and strategy of individualized operative treatment of DDH using osteotomy simulation. 


\section{Materials and methods}

Subjects. A total of 56 patients (28 females and 28 males) with DDH were recruited at the Affiliated Hongqi Hospital of Mudanjiang Medical University between May 2014 and July 2017. The mean age of the patients was 17.5 years (range, 12.0-19.5 years). DDH was diagnosed by computed tomography $(\mathrm{CT})$ imaging examination and all subjects had unilateral or bilateral DDH. The severity of DDH was defined according to three grades: Instability, subluxation and dislocation, as previously described (13). None of the patients had any history or symptoms of DDH and patients with any systemic syndrome were excluded from the study.

Processing of 3D-printed pelvis model. The 3D printing experiments were performed using a self-developed selective laser melting machine, DiMetal-100 (South China University of Technology). The 3D-printed pelvis model was directed by a scanning galvanometer according to a previous study (14).

The settings for the procedure were as follows: Scanning speed, $30-3,000 \mathrm{~mm} / \mathrm{sec}$; thickness of layer, 20-120 $\mu \mathrm{m}$; focusing spot diameter, $80 \mu \mathrm{m}$. The largest size of the part produced was $100 \times 100 \times 120 \mathrm{~mm}$.

Surgical reconstruction guided by a 3D-printed pelvis model. The osteotomy surgery was performed with the guidance of the 3D-printed pelvis model and the final reduction was pre-assessed to obtain the best-fit position for each DDH patient. The surgery was first simulated using a 3D-printed pelvis model. By using the virtual skeletal model, the optimal osteotomy position and angle were determined to achieve the best wedge resection geometry during osteotomy surgery.

Pelvic osteotomy. All operations were planned with the 3D model and performed using pelvic osteotomy as described previously (15). In brief, the hip joint of patients was approached superficially between the sartorius and tensor fascia lata and the deep dissection was between the hip abductors and rectus. The anteromedial capsule was adequately incised and the hip was identified; the ligamentum teres was cut and traced to the acetabulum. Soft-tissue release was applied in all hips; the hip was abducted and adducted, and the zone of abduction and adduction in which the femoral head remains reduced in the acetabulum was determined. The orientation of the osteotomy was marked on the lateral cortex of the ilium and a straight 0.5 -inch osteotome was used to perform the bone cut. A guide wire was inserted under fluoroscopic control at the most cephalad point of the curvilinear marking line, which was used to ensure that the osteotomy terminated at the appropriate level just above the horizontal limb of the triradiate cartilage. The osteotomy site was kept open by inserting two correctly sized bone grafts and fixed with metallic internal fixation. After the insertion of femoral and acetabular components, computed tomography was performed to evaluate the efficacy of surgery.

Evaluation. The bone and model measurements were compared to assess the accuracy of the osteotomies guided by the 3D printed model or CT-based model. Surgical time, post-operative recovery time, hospital stay and an inflammation-based prognostic score were recorded for all patients (16). CT examination
Table I. Characteristics of patients with developmental dysplasia of the hip.

\begin{tabular}{lccc}
\hline Characteristic & 3D-CT & $\begin{array}{c}\text { 3D-printed } \\
\text { pelvis model }\end{array}$ & P-value \\
\hline Total number & $38(67.9)$ & $18(32.1)$ & 0.026 \\
Sex & & & \\
$\quad$ Female & $20(52.6)$ & $8(44.4)$ & 0.054 \\
$\quad$ Male & $18(47.4)$ & $10(55.6)$ & 0.068 \\
Side affected & & & \\
$\quad$ Right & $28(73.7)$ & $8(44.4)$ & 0.022 \\
$\quad$ Left & $10(26.3)$ & $10(55.6)$ & 0.034 \\
Age (years) & $32 \pm 4$ & $36 \pm 5$ & 0.68 \\
Severity of dysplasia & & & \\
$\quad$ Instability & $10(26.3)$ & $5(27.8)$ & 0.46 \\
Subluxation & $15(39.5)$ & $7(38.9)$ & 0.52 \\
$\quad$ Dislocation & $13(34.2)$ & $6(33.3)$ & 0.76 \\
Follow-up (months) & 24 & 24 & - \\
\hline
\end{tabular}

Values are expressed as the mean \pm standard deviation, $\mathrm{n}$, or $\mathrm{n}(\%)$. CT, computed tomography.

was performed three days after the osteotomy surgery. Hip function was assessed using the Majeed score (17). The accuracy of the internal fixation was determined from the post-operative CT. The patients' hip joints were classified according to Tonnis measurements of the acetabula angles into 4 grades using International Hip Dysplasia Institute classification system as described previously (18). The detailed summary scores and the visual analog scale of satisfaction mean score were recorded in patients as described previously (19). The evaluations and follow-up (24 months) were performed by the same three independent doctors.

Statistical analysis. Values are expressed as the mean \pm standard deviation and statistical analyses were performed using SPSS version 19.0 software (IBM Corp.). Student's t-test was used to compare the measurements made on the 3D printed models to those from the 3D-CT based pelvis model. $\mathrm{P}<0.05$ was considered to indicate statistical significance.

\section{Results}

Patient characteristics. A total of $56 \mathrm{DDH}$ patients (28 female, 28 male) were included in the present study. DDH was located on the right hip in 36 cases and on the left in 20 cases. The mean follow-up time was 24 months. A total of $18 \mathrm{DDH}$ patients ( 8 female, 10 male) were treated by surgery planned by using the 3D-printed pelvis model and $38 \mathrm{DDH}$ patients (20 female, 18 male) received surgery planned by using 3D-CT. All patients were randomly recruited and voluntarily received 3D-printed pelvis model or 3D-CT. The baseline characteristics of the DDH patients are summarized in Table I. Regarding the different severities of DDH (instability, subluxation and dislocation), the percentage of male and female patients was not significantly different between the two groups. 
Table II. Efficacy of 3D-printed pelvis model for patients with developmental dysplasia of the hip.

\begin{tabular}{lccc}
\hline & \multicolumn{3}{c}{ 3D-printed } \\
Item & 3D-CT & pelvis model & P-value \\
\hline Success rate (\%) & 82.4 & 94.2 & 0.040 \\
Surgery time (h) & $6.2 \pm 1.5$ & $4.8 \pm 2.0$ & 0.026 \\
$\begin{array}{l}\text { Post-operative recovery } \\
\text { time (days) }\end{array}$ & $28.5 \pm 7.0$ & $21.0 \pm 5.0$ & 0.036 \\
Total redislocations & $5(17.8)$ & $3(7.9)$ & 0.0053 \\
Redislocations detected & $4(10.5)$ & $2(5.6)$ & 0.038 \\
after discharge & & & \\
\hline
\end{tabular}

Values are expressed as the mean \pm standard deviation or $\mathrm{n}(\%)$, unless specified otherwise. CT, computed tomography.

Table III. Efficacy of 3D-printed pelvis model for post-operative parameters in patients with developmental dysplasia of the hip.

\begin{tabular}{lccc}
\hline Item & \multicolumn{3}{c}{ 3D-printed } \\
\hline 3ospital stay (days) & $28.0 \pm 6.0$ & $23.5 \pm 4.5$ & 0.035 \\
Inflammation score & $6.0 \pm 2.0$ & $4.0 \pm 2.0$ & 0.044 \\
Delayed incision healing & $2(7.1)$ & $2(7.1)$ & $>0.05$ \\
Wound infection & $1(3.6)$ & $1(3.6)$ & $>0.05$ \\
Nonunion & $1(3.6)$ & $1(3.6)$ & $>0.05$ \\
Majeed score & $72 \pm 10$ & $70 \pm 15$ & $>0.05$ \\
\hline
\end{tabular}

Values are expressed as the mean \pm standard deviation or $\mathrm{n}(\%)$. CT, computed tomography.

Efficacy. The efficacy of the 3D-printed pelvis model was investigated in the $56 \mathrm{DDH}$ patients $(\mathrm{n}=18$ in the $3 \mathrm{D}$-printed pelvis model group; $n=38$ in the $3 \mathrm{D}-\mathrm{CT}$ group). The success rate of surgery (at month 3 ) in those DDH patients pre-evaluated with the 3D-printed pelvis model (94.2\%) was significantly higher than that in the patients pre-evaluated by 3D-CT (82.4\%). Use of the 3D-printed pelvis model decreased the surgery time (surgery + 3D-printed pelvis model, $4.8 \mathrm{~h}$; surgery + 3D-CT, $6.2 \mathrm{~h}$ ) and shortened the post-operative recovery time for DDH patients compared with that in the surgery + 3D-CT group. In addition, the 3D-printed pelvis model significantly reduced total redislocations and redislocations detected after discharge for DDH patients (Table II).

Recovery. Representative images of the 3D-CT and 3D-printed pelvis model were provided in Fig. 1. The present results revealed that use of the $3 \mathrm{D}$-printed pelvis model allowed for more rapid recovery of the DDH patients. The time of hospital stay and inflammation score were decreased in the surgery $+3 \mathrm{D}$-printed pelvis model group compared with those in the surgery $+3 \mathrm{D}$-CT group. Furthermore, few DDH patients with osteotomy simulation using the 3D-printed pelvis model or 3D-CT had delayed incision healing, wound infection or
Table IV. Comparison of summary scores (coordination score and visual analog scale of satisfaction score) between the 3D-CT and 3D-printed pelvis model groups.

\begin{tabular}{|c|c|c|c|}
\hline Parameter & 3D-CT & $\begin{array}{l}\text { 3D-printed } \\
\text { pelvis model }\end{array}$ & P-value \\
\hline $\begin{array}{l}\text { Upper limb coordination } \\
\text { score }\end{array}$ & $7.01 \pm 1.56$ & $7.12 \pm 1.38$ & 0.64 \\
\hline $\begin{array}{l}\text { Lower limb coordination } \\
\text { score }\end{array}$ & $7.52 \pm 1.69$ & $7.40 \pm 1.46$ & 0.72 \\
\hline Pelvis coordination score & $4.64 \pm 1.52$ & $6.80 \pm 1.52$ & $<0.01$ \\
\hline $\begin{array}{l}\text { Visual analog scale of } \\
\text { satisfaction }\end{array}$ & $5.36 \pm 1.21$ & $7.58 \pm 1.46$ & $<0.01$ \\
\hline
\end{tabular}

Values are expressed as the mean \pm standard deviation. CT, computed tomography.

Table V. Pre- and post-operative acetabular index and center edge angle $\left({ }^{\circ}\right)$ for patients with developmental dysplasia of the hip.

\begin{tabular}{lcc}
\hline Time-point/angle type & 3D-CT & $\begin{array}{c}\text { 3D-printed } \\
\text { pelvis model }\end{array}$ \\
\hline Prior to the operation & & \\
Acetabular index angle & $38.5 \pm 10.5$ & $40.6 \pm 12.6$ \\
Center edge angle & $14.2 \pm 6.8$ & $13.5 \pm 7.5$ \\
Post-operation (3 months) & & \\
Acetabular index angle & $22.6 \pm 4.2$ & $24.3 \pm 4.8^{\mathrm{a}}$ \\
Center edge angle & $27.5 \pm 5.6$ & $24.4 \pm 6.0^{\mathrm{a}}$ \\
\hline
\end{tabular}

${ }^{\text {a }}<0.05$ vs. $3 \mathrm{D}-\mathrm{CT}$. Values are expressed as the mean \pm standard deviation. CT, computed tomography.

nonunion. The overall Majeed score demonstrated an similar between the surgery $+3 \mathrm{D}$-printed pelvis model group and the surgery $+3 \mathrm{D}-\mathrm{CT}$ groups (Table III).

Outcomes. The detailed summary scores and the visual analog scale of satisfaction mean score are provided in Table IV. The scores for the pelvis and the spine tests for the 3D-CT group were significantly lower than those for the 3D-printed pelvis model group $(\mathrm{P}<0.01)$. However, no significant differences were observed in the scores for the upper limb test or the lower limb test $(\mathrm{P}=0.64$ and $\mathrm{P}=0.72$, respectively). The visual analog scale of satisfaction mean score was $7.49 \pm 1.38$ and $5.80 \pm 1.30$ in 3D-printed pelvis model group and 3D-CT group, respectively $(\mathrm{P}<0.01)$.

The pre- and post-operative acetabular index and center edge angle were compared between the two groups of patients. There was no significant difference in the pre-operative acetabular index $\left(38.5 \pm 10.5\right.$ vs. $\left.40.6 \pm 12.6^{\circ}\right)$ and center edge angle $\left(14.2 \pm 6.8\right.$ vs. $\left.13.5 \pm 7.5^{\circ}\right)$ between the $3 \mathrm{D}$-printed pelvis model group and the $3 \mathrm{D}-\mathrm{CT}$ group. As indicated in Table V, none of the patients in the two groups presented with any hip deformities or torticollis. 

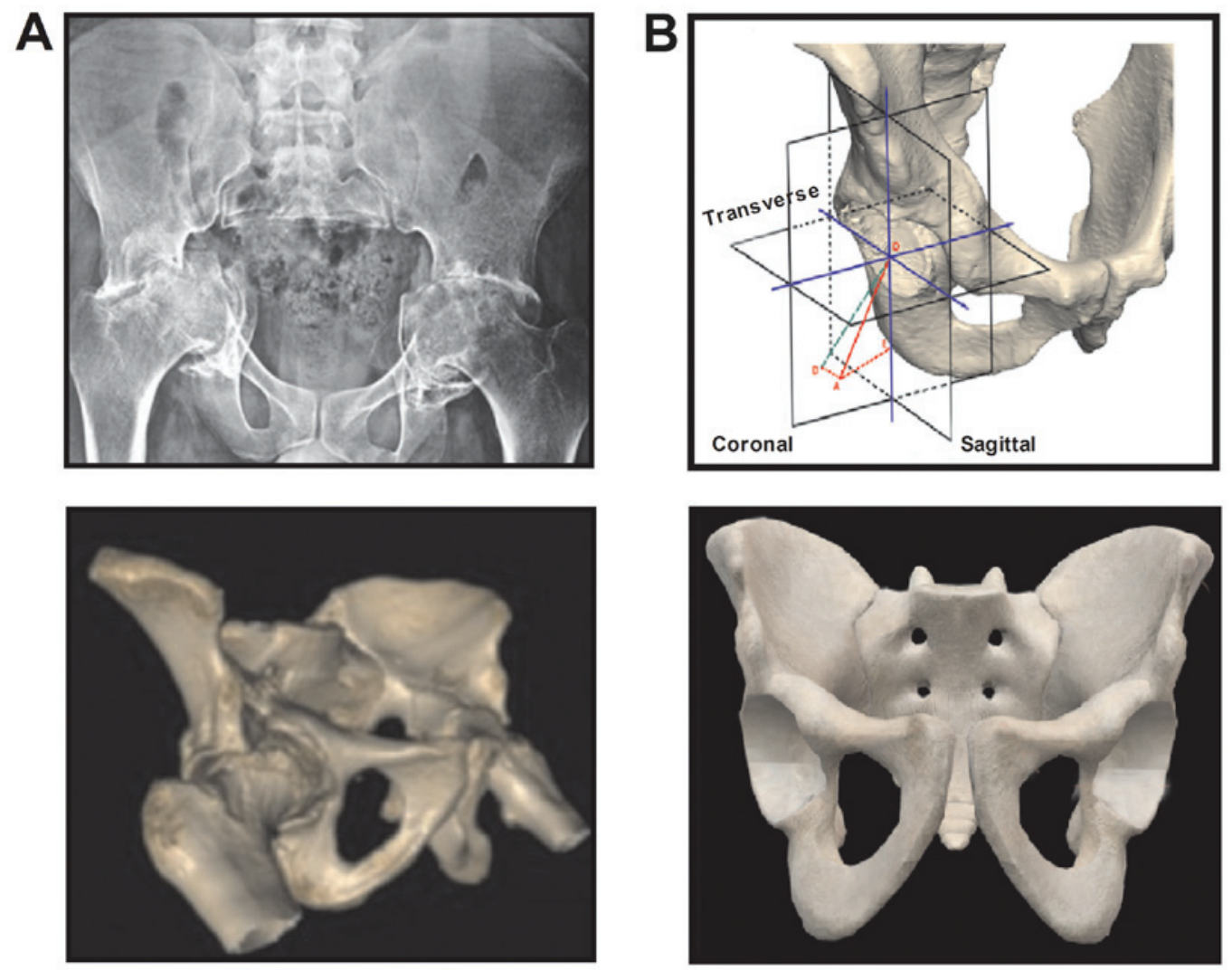

3D-CT

3D printing

Figure 1. Representative images of 3D-printed pelvis model and 3D-CT-based pelvis model (A) Representative images of 3D-CT. (B) Representative images of 3D-printed pelvis model. CT, computed tomography.

The average acetabula index at month 3 was $22.6 \pm 4.2$ and $24.3 \pm 4.8^{\circ}$ in the surgery $+3 \mathrm{D}$-printed pelvis model group and the surgery $+3 \mathrm{D}-\mathrm{CT}$ group, respectively $(\mathrm{P}<0.05)$. The average center edge angle was $27.5 \pm 5.6$ and $24.4 \pm 6.0^{\circ}$ in the surgery $+3 \mathrm{D}$-printed pelvis model group and the surgery + 3D-CT group, respectively (Table V). DDH patients in the surgery $+3 \mathrm{D}$-printed pelvis model group had a better radiographic acetabular index and center edge angle than those in the surgery $+3 \mathrm{D}-\mathrm{CT}$ group.

\section{Discussion}

With the development of 3D printing technology, its application in life science is increasing and it is becoming an important tool in medical treatments (20). A previous study has indicated that putting 3D modeling and 3D printing into practice is beneficial for virtual surgery and pre-operative planning to reconstruct complex post-traumatic skeletal deformities and defects (21). In the present study, the auxiliary efficacy of a 3D-printed pelvis model combined with surgery was investigated in a total of $38 \mathrm{DDH}$ patients with 3D-CT planning (18 DDH patients) as a control. The results indicated that the 3D-printed pelvis model comprising 3D reconstruction, reverse engineering and rapid prototyping is beneficial for osteotomy simulation in patients with DDH.

A previous study reported on the efficacy of 3D printing simulated operation and the associated improvement in the accuracy and safety of minimally invasive surgery through a small incision lateral to the rectus abdominis for pelvic fracture (22). Xiao et al (23) demonstrated that 3D printing technology increased the predictability, feasibility and reliability of simultaneous mandibular contour osteoplasty and orthognathic surgery. The present study indicated that a 3D-printed pelvis model improved the accuracy of pelvic osteotomy, and also decreased the surgery time and post-operative inflammation compared to 3D-CT-based surgery for patients with DDH. In a previous study, dimensional evaluation of patient-specific 3D printing using calcium phosphate cement provided a good degree of fitting and accuracy for craniofacial bone reconstruction (24). In the present study, the 3D-printed pelvis model was able to accurately represent the morphology and various angles of the pelvis using osteotomy simulation. Furthermore, through the use of rapid prototyping, 3D printing models have been used in orthopaedic surgery, which offers surgeons a number of advantages when treating complex fractures (14). The present study indicated that use of the 3D-printed pelvis model increased the accuracy of the surgery and decreased the time of surgery, which led to a higher success rate of the operation and shorter post-operative recovery time for DDH patients.

The acetabula index is considered the most reliable radiographic measure to evaluate the development of DDH (25). The average acetabular index was significantly improved in patients with DDH receiving 3D-printed pelvis model-based surgery compared with those treated by 3D-CT-based surgery 
with an upper limit of normal of $<30^{\circ}$. Radiological post-operative assessment of the center edge angle remains the primary factor influencing normal hip development and is required to optimize the development of the hip with the minimum number of operations (26). In the present study, the center edge angle was markedly improved in the $3 \mathrm{D}$-printed pelvis model-based surgery group compared with that in the 3D-CT-based surgery group. Good follow-up results were achieved, with no evidence of loosening of the acetabular components, or any type of hip deformity or torticollis.

The limitation of the study is that it was a retrospective preliminary study, and the number of patients was relatively small in both the 3D-printed pelvis model and 3D-CT groups. In addition, the follow-up for all DDH patients was relatively short. Furthermore, the data of the present study were from a single medical center and the full post-operative radiological information was not available for all patients. Future studies should include a large number of patients with DDH and also evaluate the cost of treatment of this disease between the $3 \mathrm{D}$-printed pelvis model and the 3D-CT group.

In conclusion, in the present study, the potential application of the 3D-printed pelvis model for DDH was investigated. The results indicated that the 3D-printed pelvis model may serve as an important tool for the individualized treatment of DDH patients. Further research is required to validate the application of the 3D-printed pelvis model for DDH therapy.

\section{Acknowledgements}

Not applicable.

\section{Funding}

No funding was received.

\section{Availability of data and materials}

The datasets used and/or analyzed during the current study are available from the corresponding author on reasonable request.

\section{Authors' contributions}

KXL, ZTL, and YBM performed the experiments, analyzed the data and conducted the statistical analysis. HYL designed the experiments, wrote the original manuscript and revised the manuscript.

\section{Ethics approval and consent to participate}

The present study was approved by the Ethics Committee of Mudanjiang Medical University (Mudanjiang). Written informed consent was obtained from all participants.

\section{Patient consent for publication}

Not applicable.

\section{Competing interests}

The authors declare that they have no competing interests.

\section{References}

1. Toma P, Valle M, Rossi U and Brunenghi GM: Paediatric hip-ultrasound screening for developmental dysplasia of the hip: A review. Eur J Ultrasound 14: 45-55, 2001.

2. Yang S and Cui Q: Total hip arthroplasty in developmental dysplasia of the hip: Review of anatomy, techniques and outcomes. World J Orthop 3: 42-48, 2012.

3. Rhodes AM and Clarke NM: A review of environmental factors implicated in human developmental dysplasia of the hip. J Child Orthop 8: 375-379, 2014.

4. Canavese F, Vargas-Barreto B, Kaelin A and de Coulon G: Onset of developmental dysplasia of the hip during clubfoot treatment: Report of two cases and review of patients with both deformities followed at a single institution. J Pediatric Orthop B 20: 152-156, 2011.

5. Gardner RO, Bradley CS, Howard A, Narayanan UG, Wedge JH and Kelley SP: The incidence of avascular necrosis and the radiographic outcome following medial open reduction in children with developmental dysplasia of the hip: A systematic review. Bone Joint J 96-B: 279-286, 2014.

6. Di Mascio L, Carey-Smith R and Tucker K: Open reduction of developmental hip dysplasia using a medial approach: A review of 24 hips. Acta Orthop Belg 74: 343-348, 2008.

7. Eley KA, Watt-Smith SR and Golding SJ: 'Black Bone' MRI: A novel imaging technique for 3D printing. Dentomaxillofac Radiol 46: 20160407, 2017.

8. Zhou Z, Buchanan F, Mitchell C and Dunne N: Printability of calcium phosphate: Calcium sulfate powders for the application of tissue engineered bone scaffolds using the 3D printing technique. Mater Sci Eng C Mater Biol Appl 38: 1-10, 2014.

9. Zheng P, Xu P, Yao Q, Tang K and Lou Y: 3D-printed navigation template in proximal femoral osteotomy for older children with developmental dysplasia of the hip. Sci Rep 7: 44993, 2017.

10. Wu XB, Wang JQ, Zhao CP, Sun X, Shi Y, Zhang ZA, Li YN and Wang MY: Printed three-dimensional anatomic templates for virtual preoperative planning before reconstruction of old pelvic injuries: Initial results. Chin Med J (Engl) 128: 477-482, 2015.

11. Zengy Y, Min L, Lai OJ, Shen B, Yang J, Zhou ZK, Kang PD and Pei FX: Acetabular morphological analysis in patients with high dislocated DDH using three-dimensional surface reconstruction technique. Sichuan Da Xue Xue Bao Yi Xue Ban 46: 296-300, 2015 (In Chinese).

12. Xu J, Li D, Ma RF, Barden B and Ding Y: Application of rapid prototyping pelvic model for patients with DDH to facilitate arthroplasty planning: A pilot study. J Arthroplasty 30: 1963-1970, 2015.

13. Dai J, Shi D, Zhu P, Qin J, Ni H, Xu Y, Yao C, Zhu L, Zhu H, Zhao B, et al: Association of a single nucleotide polymorphism in growth differentiate factor 5 with congenital dysplasia of the hip: A case-control study. Arthritis Res Ther 10: R126, 2008.

14. Upex P, Jouffroy P and Riouallon G: Application of 3D printing for treating fractures of both columns of the acetabulum: Benefit of pre-contouring plates on the mirrored healthy pelvis. Orthop Traumatol Surg Res 103: 331-334, 2017.

15. Tuhanioglu U, Cicek H, Ogur HU, Seyfettinoglu F and Kapukaya A: Evaluation of late redislocation in patients who underwent open reduction and pelvic osteotomy as treament for developmental dysplasia of the hip. Hip Int 28: 309-314, 2018

16. Watt DG, McSorley ST, Park JH, Horgan PG and McMillan DC: A postoperative systemic inflammation score predicts short- and long-term outcomes in patients undergoing surgery for colorectal cancer. Ann Surg Oncol 24: 1100-1109, 2017.

17. Bajada S and Mohanty K: Psychometric properties including reliability, validity and responsiveness of the Majeed pelvic score in patients with chronic sacroiliac joint pain. Eur Spine J 25: 1939-1944, 2016.

18. Miao M, Cai H, Hu L and Wang Z: Retrospective observational study comparing the international hip dysplasia institute classification with the Tonnis classification of developmental dysplasia of the hip. Medicine (Baltimore) 96: e5902, 2017.

19. Chandrasekaran S, Gui C, Walsh JP, Lodhia P, Suarez-Ahedo C and Domb BG: Correlation between Changes in visual analog scale and patient-reported outcome scores and patient satisfaction after hip arthroscopic surgery. Orthop J Sports Med 5: 2325967117724772, 2017.

20. Lee N: The lancet technology: 3D printing for instruments, models, and organs? Lancet 388: 1368, 2016. 
21. Tetsworth K, Block S and Glatt V: Putting 3D modelling and 3D printing into practice: Virtual surgery and preoperative planning to reconstruct complex post-traumatic skeletal deformities and defects. SICOT J 3: 16, 2017.

22. Zeng CJ, Tan XY, Huang HJ, Huang WQ, Li T, Jin DD, Zhang GD and Huang WH: Clincial effect of 3D printing-assisted minimal invasive surgery through a small incision lateral to the rectus abdominis for pelvic fracture. Nan Fang Yi Ke Da Xue Xue Bao 36: 220-225, 2016 (In Chinese).

23. Xiao Y, Sun X, Wang L, Zhang Y, Chen K and Wu G: The application of 3D printing technology for simultaneous orthognathic surgery and mandibular contour osteoplasty in the treatment of craniofacial deformities. Aesthetic Plast Surg 41: 1413-1424, 2017.
24. Bertol LS, Schabbach R and Dos Santos LAL: Dimensional evaluation of patient-specific 3D printing using calcium phosphate cement for craniofacial bone reconstruction. J Biomater Appl 31: 799-806, 2017.

25. Nie Y, Wang H, Huang Z, Shen B, Kraus VB and Zhou Z: Radiographic underestimation of in vivo cup coverage provided by total Hip arthroplasty for dysplasia. Orthopedics 41: e46-e51, 2018.

26. Murphy RF and Kim YJ: Surgical Management of pediatric developmental dysplasia of the Hip. J Am Acad Orthop Surg 24: 615-624, 2016

(i) $(-)$ This work is licensed under a Creative Commons Attribution-NonCommercial-NoDerivatives 4.0 International (CC BY-NC-ND 4.0) License. 